\title{
Au-delà de la littérature beur?, dossier coordonné par Alec G. Hargraves et Anne Marie Gans-Guinoune
}

\author{
Ilaria Vitali
}

\section{(2) OpenEdition}

1 Journals

\section{Edizione digitale}

URL: http://journals.openedition.org/studifrancesi/6119

DOI: 10.4000/studifrancesi.6119

ISSN: 2421-5856

\section{Editore}

Rosenberg \& Sellier

\section{Edizione cartacea}

Data di pubblicazione: 1 mai 2011

Paginazione: 220-221

ISSN: 0039-2944

\section{Notizia bibliografica digitale}

Ilaria Vitali, «Au-delà de la littérature beur?, dossier coordonné par Alec G. Hargraves et Anne Marie

Gans-Guinoune», Studi Francesi [Online], 163 (LV | I) | 2011, online dal 30 novembre 2015, consultato il 10 janvier 2021. URL: http://journals.openedition.org/studifrancesi/6119 ; DOI: https://doi.org/

10.4000/studifrancesi.6119

Questo documento è stato generato automaticamente il 10 janvier 2021.

\section{(c)}

Studi Francesi è distribuita con Licenza Creative Commons Attribuzione - Non commerciale - Non opere derivate 4.0 Internazionale. 


\title{
Au-delà de la littérature beur?, dossier coordonné par Alec G. Hargraves et Anne Marie Gans-Guinoune
}

\author{
Ilaria Vitali
}

\section{NOTIZIA}

Au-delà de la littérature beur?, dossier coordonné par Alec G. HARGRAVES et Anne Marie GANS GUINOUNE, «Expressions maghrébines», vol. 7, n. 1, estate 2008, pp. 248.

1 Tutti i critici che si sono avvicinati alla letteratura beur hanno dovuto fare i conti con la difficoltà di definizione che l'accompagna. Una difficoltà che viene dal fatto che questo «rejeton hexagonal de la littérature maghrébine» (Keil, 1991) mostra di avere sin dall'inizio qualcosa di diverso rispetto alla letteratura maghrebina così come rispetto a quella francese, situandosi in un entre-deux difficile da identificare e da descrivere. Secondo uno dei maggiori studiosi di letteratura beur, Alec G. Hargreaves: «La littérature issue de l'immigration en France est une littérature qui gêne. Les documentalistes ne savent pas où la classer, les enseignants hésitent à l'incorporer dans leurs cours et les critiques sont généralement sceptiques quant à ses mérites esthétiques» (Hargreaves, 1996). In questo numero tematico di «Expressions maghrébines», è proprio Alec G. Hargreaves, insieme ad Anne Marie Gans-Guinoune, ad invitare ad andare, come recita il titolo, «au-delà de la littérature beur», puntando $\mathrm{i}$ riflettori verso nuovi scritti e nuovi approcci critici. I contributi raccolti nel volume si propongono infatti di fare il punto della situazione attuale, a trent'anni dalla nascita della letteratura beur, soffermandosi in particolare sugli autori della nuova generazione, come Faïza Guène e Mohamed Razane.

2 Nell'introduzione, i curatori tracciano il percorso dell'etichetta beur, nata nei primi anni Ottanta in seguito a diversi movimenti pacifisti e antirazzisti («La Marche pour l'égalité et contre le racisme», "Convergences $84 » . .$.$) , e successivamente applicata a$ 
varie espressioni culturali del mondo beur, tra cui la letteratura. Sulla pertinenza di tale definizione, tuttavia, sono apparsi fin dal principio non pochi dubbi da parte degli studiosi, che si sono chiesti se il termine, carico di connotazioni extra-letterarie, non fosse troppo discriminante. L'insoddisfazione verso l'uso di questo label, ha portato a diversi altri tentativi, più o meno riusciti, di definizione. Littérature française d'expression immigrée, littérature franco-maghrébine, littérature arabo-française, littérature décentrée, littérature issue de l'immigration maghrébine, littérature de la deuxième génération... sono solo alcune delle alternative proposte. A trent'anni dalla nascita di questa letteratura, l'ansia tassonomica non si è calmata, anzi, abbondano le nuove etichette, nessuna delle quali ha però trovato un uso condiviso.

3 Nell'articolo che apre la raccolta, Passé oublié, passé regagné (pp. 13-31), Crystel PINÇONNAT riprende il delicato dibattito sulle etichette e traccia poi un rapido percorso della letteratura in questione, dalle origini ai giorni nostri, soffermandosi sugli aspetti tematici. Se i primi testi letterari beurs appaiono nei primissimi anni Ottanta (Hocine Touabti, L'amour quand même, 1981; Mohamed Kenzi, Temps maure, 1981), sono due i romanzi che segnano particolarmente la scena culturale francese di quegli anni: Le thé au harem d'Archi Ahmed di Mehdi Charef (1983) - comunemente ritenuto dalla critica punto d'inizio della letteratura beur - e Le gone du Chaâba (1986) di Azouz Begag, che porta ad un «consolidamento» dell'etichetta beur. Dagli anni Ottanta in poi, sono molti gli autori beurs che si sono affacciati sulla scena letteraria francese. Come ricorda Hargreaves nella rassegna critica che chiude il volume, si nota infatti un aumento costante nella produzione e pubblicazione di testi. Per Pinçonnat, dopo essersi a lungo concentrati su temi come la banlieue, la «galère» e «le monde des cousins», la letteratura beur riscopre oggi la memoria dell'immigrazione e, soprattutto, la delicata questione identitaria, concentrandosi sull'eredità di tradizioni diverse, il cui incontro è conflittuale ma quanto mai produttivo. L'articolo di Dominic THOMAs ha il compito di «traghettare» il lettore verso le scritture del nuovo millennio. Oggetto dell'analisi, dal titolo New writings for New Times (pp. 33-51), è l'opera di Faïza Guène, una delle autrici più promettenti di quella che Thomas definisce "generazione post-beur». L'autore si sofferma sulla banlieue, non concepita soltanto come tema o sfondo letterario, ma come fenomeno sociologico e principio estetico. Thomas sottolinea a tal proposito l'apporto del collettivo di scrittori «Qui fait la France?», a cui la stessa Guène appartiene, nella creazione di un imperativo impegno sociale, sostenuto da un vero e proprio manifesto (si veda <www.quifaitlafrance.com>).

4 Anche Patricia GEESEY si concentra sull'analisi dell'opera di Faïza Guène, una delle autrici beurs del contemporaneo estremo che godono di maggior successo e diffusione. Nell'articolo Global Pop Culture in Faïza Guène's "Kiffe kiffe demani" (pp. 53-66), l'autrice esamina il romanzo d'esordio di Guène, best-seller pluritradotto, soffermandosi in particolare sulle numerose influenze della cultura pop globale sulla rappresentazione del mondo contemporaneo dei margini francesi. Nell'articolo successivo, Laura K. REECK (Mohamed Razane: The Re-generation of Beur literature, pp. 67-83) sonda invece l'opera di un altro membro del collettivo «Qui fait la France?»: Mohamed Razane. La studiosa vi sottolinea il ruolo guida dell'autore - fondatore e presidente del collettivo - dopo le rivolte scoppiate in banlieue nel 2005. Il romanzo di Razane, Dit violent (2006), è per Reeck un'esplorazione delle origini, dello sviluppo e della legittimazione della violenza, questioni delicate che assumono una sfumatura particolare se applicate alle banlieue francesi. Segue un'intervista di Steve Puig allo stesso Razane (pp. 85-92), in cui 
emergono diversi punti-chiave del pensiero dell'autore, in particolare il rifiuto dell'etichetta «beur», la volontà estrema di realismo e l'idea - ripresa da Stendhal - che la letteratura sia uno specchio da portarsi dietro per le strade, in particolare quelle di banlieue. L'articolo successivo, L'effet beur (pp. 93-107), si concentra sulla ricezione giornalistica della letteratura in questione. Kenneth oLsson vi indaga, attraverso l'analisi di quindici romanzi beurs pubblicati tra il 2005 e il 2006, la prospettiva adottata di volta in volta da giornalisti e commentatori televisivi. Ad emergere nel processo di ricezione mediatica è, come è facile immaginare, un'influenza diretta di fatti di cronaca e fenomeni sociali di grande portata, come le rivolte in banlieue del novembre 2005.

5 La letteratura beur è dunque inevitabilmente destinata a «occuper les banlieues ou les ZUP de la littérature» (Begag e Chaouite)? A interrogarsi a questo proposito è anche Faroud LAROUSSI, che, nell'articolo La littérature «beur» ou le paradoxe de l'authenticité (pp. 109-120), mette in luce i rischi dell'autenticità ricercata come un «marchio di fabbrica». In tal senso, una prospettiva interessante è quella adottata da Myriam GEISER nell'articolo La «littérature beur» comme écriture de la post-migration et forme de «littératuremonde» (pp. 121-139). Per evitare le connotazioni extra-letterarie del termine beur, l'autrice propone qui l'etichetta "littérature de la post-migration», concetto che, secondo Geiser, permette di prescindere dalla classificazione etnica e di stabilire una relazione con il fenomeno della «littérature-monde». La letteratura beur è infatti per Geiser basata sul concetto di métissage culturel, non interpretabile dunque secondo una griglia puramente nazionale.

Nell'articolo The Algerian War and its Afterlives Painted Noir in "Sérail Killers" by Lakhdar Belaïd (pp. 142-157), Sylvie DURMELAT analizza il romanzo d'esordio di Belaïd, in bilico tra romanzo beur e polar. Lo studio mette in luce, in particolare, come la rappresentazione della Guerra d'Algeria sia notevolmente cambiata rispetto ai romanzi maghrebini degli anni Sessanta. L'articolo di Michèle BACHOLLE-BošKovic, Et les enfants, alors? Une littérature beur de jeunesse? (pp. 159-176), tocca un altro punto importante della letteratura beur: la presenza di numerosi romanzi destinati ai ragazzi. Diversi autori beurs hanno infatti contribuito alla narrativa dedicata ai più piccoli (per esempio, Azouz Begag), rientrando, in questo senso, nel «canone» della littérature française de jeunesse. L'ultimo articolo di questo numero è quello di Anissa TALAHITEMOODLEY, che analizza Deux romans de la rupture et du renouvellement: "Le Marteau piquecoeur" de Azouz Begag et "Le Dromadaire de Bonaparte" de Tassadit Imache (pp. 177-192). L'autrice mette in evidenza come i personaggi dei romanzi di Begag e di Imache rappresentino un netto rifiuto di ogni identità precostituita e irrevocabile.

7 Chiude questa ricca raccolta di studi un inventario aggiornato degli autori beurs dalle origini ai nostri giorni, realizzato da Alec G. HARGREAVES, già autore degli unici repertori presenti a tutt'oggi (La littérature beur: un guide bio-bibliographique, 1992; Voices from the North African Immigrant Community in France: Immigration and Identity in Beur Fiction, 1997). Ad emergere da questa rassegna bibliografica è soprattutto la mole di testi, in continuo aumento (anche grazie ad un rinnovato interesse editoriale), che rende ormai difficile considerare la letteratura beur un fenomeno marginale. Ad arricchire la riflessione critica, la sezione "Création» accoglie alcuni stralci di un romanzo di Leyla TATZBER, dal titolo Tache de beur, poi pubblicato nel 2008 da L'Hèbe. La sezione Varia ospita invece un interessante articolo di Najib EL JABBAR, Abdelkébir Khatibi. Désir d'oubli, désir de mémoire, dedicato all'opera del grande scrittore marocchino. 
Colpisce, in questo volume, la continua riflessione, quasi ossessiva, sulla definizione di letteratura «beur», la cui problematicità rischia, a tratti, di accaparrare l'attenzione della critica ben più dei testi degli autori. Pur con qualche rischio e limite (quale etichetta non ne ha?), il label beur rimane ancor oggi, per molti versi, il più preciso nel definire la letteratura in questione. In esso traspaiono infatti, come già aveva sottolineato Keil, tre elementi caratterizzanti: l'arabité (beur è, com'è noto, il verlan della parola «arabe»), la francité (il verlan è un procedimento di formazione lessicale tipicamente francese), la marginalité (il verlan è il linguaggio caratteristico delle banlieue). Sono tuttavia numerose, come si è visto, le nuove etichette proposte dalla critica. Una tendenza affermatasi negli ultimi anni ha visto la nascita del label «letteratura di banlieue», definizione più inglobante, in cui rientrerebbero non soltanto gli autori beurs - sebbene questi rimangano una forte maggioranza - ma tutti gli autori che parlano dai/dei margini toute origine confondue. Ne costituiscono l'emblema i membri del collettivo "Qui fait la France?», composto da dieci autori della banlieue parigina, che si vuole una rappresentazione della Francia multietnica. C'è dunque da parte degli autori dell'ultima generazione una volontà di trascendere il mondo beur, anche se, di fatto, i membri di questo collettivo che si propone come una rappresentazione realistica della Francia contemporanea sono in realtà per la maggior parte di origine maghrebina ( 7 su 10). La tendenza è tuttavia evidente: se in un primo tempo molti autori si erano riconosciuti nell'etichetta di «letteratura beur» o di «letteratura di banlieue», oggi la rifiutano e chiedono di essere scrittori tout court. È tuttavia paradossale che, nonostante vogliano smarcarsi da definizioni che trovano riduttive e ghettizzanti, continuino spesso nei loro romanzi a riprodurre stereotipi che non fanno che collocarli in modo sempre più netto all'interno di quelle etichette (con buona responsabilità del circuito editoriale). Insomma, la questione, delicata $\mathrm{e}$ complessa, rimane aperta. E la singolare voce di questa letteratura, la sua forza e la sua ricchezza stanno forse proprio in questa sua problematicità. 